\title{
Aspirin use and bleeding volume in skin cancer patients undergoing surgery: a randomized controlled trial
}

\author{
Arman Engheta', Shahryar Hadadi Abianeh ${ }^{2 *}$, Ali Atri $^{1}$ and Mehdi Sanatkarfar ${ }^{3}$
}

\begin{abstract}
We investigated the occurrence of bleeding complications in patients who underwent skin tumor surgery and compared it between Aspirin users and a placebo control group. In this double blind randomized controlled trial, 32 patients who continued taking aspirin (intervention group) and 38 patients who stopped taking Aspirin (placebo group) before surgery were compared in terms of intraoprative and postoperative bleeding problems, hematoma and local signs of coagulopathy. There was no statistically significant difference in intraoprative bleeding between the study groups $(P=0.107)$. We concluded that continuation of Aspirin therapy had no significant effect on bleeding complications in patients who underwent skin tumor surgery.
\end{abstract}

Trial registration: IRCT201602049768N5

Keywords: Acetyl salicylic acid, Skin cancer, Surgery, Bleeding, Complication, Aspirin

Abbreviations: BT, Bleeding time; BUN, Blood urea nitrogen; CT, Clotting time; FBS, Fasting blood sugar; INR, International normalized ratio; PT, Prothrombin time; PTT, Partial thromboplastin time; SPSS, Statistical Package for the Social Sciences

Discontinuation of anticoagulant or anti platelet agents before skin surgery is still a challenge due to the lack of proper recommendations in the current guidelines [1]. For the decision making, the surgeon should consider several patient-related factors, such as indication of the treatment, patient's condition and the underlying disease, in order to decide about the continuation or interruption of the drug $[2,3]$. Skin surgeries are considered as one of the safest and simplest surgeries. However, rapid increase in the use and new indications of anticoagulant drugs, particularly aspirin, requires specific attention toward their use in skin surgeries $[4,5]$.

However, the evidence regarding the continuation or discontinuation of Aspirin before skin surgery is inconsistent. In the present study, we aimed to monitor the bleeding complications in patients who underwent skin

\footnotetext{
* Correspondence: H_abianeh@yahoo.com

${ }^{2}$ Department of Plastic Surgery, Razi Hospital, Tehran University of Medical

Sciences, Vahdat Eslami st, Tehran, Iran

Full list of author information is available at the end of the article
}

tumor surgery and compared it between Aspirin users and a placebo control group.

In this double-blind randomized controlled trial, we enrolled patients with non-bleeding skin tumors who were under treatment with aspirin due to any indication. The inclusion criteria were use of Aspirin for at least 3 months before surgery with a daily dose of $80 \mathrm{mg}$, age between 40 and 75 years, giving an informed consent for taking part in the study, and international normalized ratio (INR) of 1-1.5. Our exclusion criteria included as follows; Having dementia, movement disorder, simultaneous participation in another trial, patients with life-threatening cardiovascular diseases (i.e. New York Heart Association class III or more, history of previous myocardial infarction, severe heart valve disease), bleeding disorders, use of antiplatelets other than Aspirin or anticoagulants and positive history of gastrointestinal bleeding. Moreover, patients who did not follow the prescription rules, those who had a disease that required Aspirin discontinuation or Aspirin intolerance were also excluded. In order to make sure about the 
drug compliance of the patients, they were asked to bring the blister pack of the consumed tablets.

Using block randomization, patients were randomized into intervention and control groups, matched for age and sex. Both groups were asked to discontinue their Aspirin 7 days before the surgery and they received packed drugs of the trial including Aspirin $(80 \mathrm{mg})$ for the intervention group and placebo for the control group.

Before operation, demographic and baseline clinical characteristics were collected from the patients. The clinical data included the presence of bleeding risk factors, type of skin tumor, number and size of the tumor(s), location of the lesion, drug history and blood test. For every patient, standard resection for the tumor was performed regarding its size and other clinical characteristics. Type of operation and data regarding anesthesia, cautery, need for osteotomy and other surgical characteristics were recorded for the patient. We measured the bleeding by weighing the dressing gases during and after operation up to $24 \mathrm{~h}$. The nurse who was in charge of weighing the gases was blinded to the study protocol. Primary endpoint of the study was the amount of bleeding within and early after surgery. Secondary endpoints were need for early changing of the dressing, development of hematoma or local anticoagulation disorders such as petechia or ecchymosis.

Table 1 Comparison of the baseline characteristics between the study groups

\begin{tabular}{llll}
\hline Characteristic $^{a}$ & $\begin{array}{l}\text { Intervention } \\
(n=32)\end{array}$ & $\begin{array}{l}\text { Placebo } \\
(n=38)\end{array}$ & $P$-value \\
\hline Age, year & $65.8 \pm 2.3$ & $64.1 \pm 1.7$ & 0.218 \\
Male gender, $n$ (\%) & $24(75)$ & $29(76.3)$ & 0.683 \\
Diabetes, $n$ (\%) & $13(40.6)$ & $5(13.2)$ & 0.009 \\
Hypertension, $n$ (\%) & $23(71.9)$ & $19(50)$ & 0.063 \\
Cardiovascular diseases, $n(\%)$ & $21(65.6)$ & $11(28.9)$ & 0.002 \\
Smoking, $n$ (\%) & $4(12.5)$ & $5(13.2)$ & 0.999 \\
Opium abuse, $n$ (\%) & $3(9.4)$ & $4(10.5)$ & 0.999 \\
FBS, mg/dl & $123.9 \pm 58.0$ & $121.7 \pm 44.9$ & 0.696 \\
BUN, mg/dl & $37.3 \pm 8.9$ & $33.7 \pm 8.7$ & 0.064 \\
Creatinine, mg/dl & $0.94 \pm 0.24$ & $0.95 \pm 0.25$ & 0.723 \\
Hemoglobin, g/dl & $13.7 \pm 1.3$ & $14.9 \pm 4.4$ & 0.054 \\
Platelet, 1/mm ${ }^{3}$ & $208.4 \pm 81.9$ & $200.3 \pm 45.1$ & 0.925 \\
INR & $2.1 \pm 4.7$ & $1 \pm 0.01$ & 0.096 \\
PT, sec & $13.3 \pm 3.2$ & $12.9 \pm 2.3$ & 0.001 \\
PTT, sec & $28.1 \pm 4.1$ & $28.9 \pm 3.2$ & 0.114 \\
CT, sec & $327.3 \pm 72.2$ & $321.7 \pm 62.9$ & 0.669 \\
BT, sec & $152.7 \pm 59.7$ & $148.7 \pm 44.6$ & 0.791 \\
\hline BT B
\end{tabular}

$B T$ Bleeding time, BUN Blood urea nitrogen, $C T$ clotting time, FBS Fasting blood sugar, INR International normalized ratio, PT Prothrombin time, PTT Partial thromboplastin time

${ }^{a}$ Variables are shown as mean \pm standard deviation or frequency (percentage) where appropriate

$\dagger P<0.05$ was considered as statistically significant
Categorical variables were analyzed by the chi-square test. Continuous variables are presented as means \pm standard deviation, or as median and interquartile ranges, as appropriate. Differences between groups in normally and nonnormal continuous variables were assessed using the unpaired Student's $t$ test and the Mann-Whitney U test,

Table 2 Comparison of the tumoral and operative characteristics between the study groups

\begin{tabular}{|c|c|c|c|}
\hline Characteristic $^{a}$ & $\begin{array}{l}\text { Intervention } \\
(n=32)\end{array}$ & $\begin{array}{l}\text { Placebo } \\
(n=38)\end{array}$ & $P$-value \\
\hline Location & & & 0.908 \\
\hline Face & $10(29.4)$ & $13(30.9)$ & \\
\hline Nose & $6(17.6)$ & $4(9.5)$ & \\
\hline Ear & $4(11.7)$ & $5(11.9)$ & \\
\hline Neck & $0(0)$ & $192.3)$ & \\
\hline Scalp & $9(26.4)$ & $14(33.3)$ & \\
\hline Other & $5(14.7)$ & $5(11.9)$ & \\
\hline Type & & & 0.675 \\
\hline Basal cell carcinoma & $24(75)$ & $32(84.2)$ & \\
\hline Squamous cell carcinoma & $5(15.6)$ & $5(13.2)$ & \\
\hline Melanoma & $1(3.1)$ & $0(0)$ & \\
\hline Not reported & $2(6.2)$ & $1(2.6)$ & \\
\hline Size of lesion & & & 0.17 \\
\hline$<3 \mathrm{~cm}$ & $15(46.9)$ & $14(35)$ & \\
\hline $3-6 \mathrm{~cm}$ & $15(46.9)$ & $24(60)$ & \\
\hline$>6 \mathrm{~cm}$ & $1(3.1)$ & $2(5)$ & \\
\hline Not reported & $1(3.1)$ & $0(0)$ & \\
\hline Number of lesions & & & 0.478 \\
\hline 1 lesion & $22(68.8)$ & $29(76.3)$ & \\
\hline 2 lesions & $3(9.4)$ & $6(15.8)$ & \\
\hline 3 lesions & $2(6.2)$ & $1(2.6)$ & \\
\hline 4 lesions and more & $3(9.4)$ & $1(2.6)$ & \\
\hline Not reported & $2(6.2)$ & $1(2.6)$ & \\
\hline Type of surgery & & & 0.72 \\
\hline Flap & $24(70.6)$ & $28(68.3)$ & \\
\hline Graft & $9(56.4)$ & $13(31.7)$ & \\
\hline Other & $1(2.9)$ & $0(0)$ & \\
\hline Type of anesthesia & & & 0.999 \\
\hline Sedative & $31(96.9)$ & $38(100)$ & \\
\hline Not reported & $1(3.1)$ & $0(0)$ & \\
\hline Cautery & & & 0.999 \\
\hline Monopolar & $31(96.9)$ & $37(97.4)$ & \\
\hline Bipolar & $1(3.1)$ & $1(2.6)$ & \\
\hline Need for osteotomy & $0(0)$ & $1(2.6)$ & \\
\hline Volume of bleeding, ml & $30[20,80]$ & $30[17,40]$ & 0.107 \\
\hline
\end{tabular}

${ }^{\mathrm{a}}$ Variables are shown as frequency (percentage) or median [interquartile range] where appropriate

$+P<0.05$ was considered as statistically significant 
respectively. All probability values were two-tailed and a $P$ value $<0.05$ was considered significant. Data were analyzed with Statistical Package for the Social Sciences (SPSS) for Windows, version 15.0 (SPSS Inc., Chicago, Ilinois, United States of America).

In the present study, 38 patients were randomized to the intervention group and 38 patients were included in the control group. However, after randomization it was revealed that three patients had used antiplatelet or anticoagulant drugs and three other patients refused to continue the study; so, they were excluded from the final analysis $(n=32$ for the intervention group). The frequency of diabetes and cardiovascular disease was significantly higher in the intervention group ( $P=0.009$ and $P=0.002$, respectively). Details of the demographic and baseline clinical characteristics of the study groups are shown in Table 1.

Based on the pathology report, characteristics of the tumors and operation were comparable between the two groups as shown in Table 2.

Bleeding in all participants was restricted to the operation time and none of the participants had postoperative bleeding. Median volume of bleeding was 30 gram in both groups $(P=0.107)$ (Table 2$)$. None of the patients required early change of wound dressing and we observed no case of hematoma or local coagulation disorder.

We found no significant difference between patients who used Aspirin perioperatively and those who discontinued it beforehand. This finding is in line with similar previous studies [6-8], while the strength of our study is its randomized controlled trial design and its uniform population that consisted of skin cancer patients. We also observed no complication within the study period.

Based on our findings, perioperative Aspirin therapy had no significant effect on bleeding complications in patients who underwent skin tumor surgery. Currently, surgical bleedings can be controlled easily by electrocauterization and are not potentially life-threatening. It seems that dermasurgeons should be more informed about the safety of Aspirin use in skin surgeries based on the current body of knowledge. Larger studies can also contribute to the elucidation of the use of multiple antiplatelet and anticoagulant agents during skin surgeries.

\section{Acknowledgements}

This study was supported by Razi Hospital and Tehran University of Medical Sciences, Tehran, Iran.

\section{Authors' contribution}

Study design: SHA, AA. Randomization, Data collection: AE. Statistical Analysis and drafting: AE, MS. Manuscript revision and final approval: All authors.

\section{Competing interests}

The authors declare that they have no competing interests.

\section{Declarations}

All participants signed a written informed consent. The protocol of this study was approved by the institutional ethical committee and board of research and is in accordance with the Declaration of Helsinki.
All the authors have read the article and gave consent for its publication as a letter to editor.

Data and material of this study is available via the corresponding author.

This work was funded by Tehran University of Medical Sciences.

\section{Author details}

${ }^{1}$ Department of Plastic Surgery, Imam Khomeini Hospital, Tehran University of Medical Sciences, Tehran, Iran. ${ }^{2}$ Department of Plastic Surgery, Razi Hospital, Tehran University of Medical Sciences, Vahdat Eslami st, Tehran, Iran. ${ }^{3}$ Department of Anesthesiology, Razi Hospital, Tehran University of Medical Sciences, Tehran, Iran.

Received: 28 May 2016 Accepted: 12 July 2016

Published online: 28 July 2016

References

1. Alam M, Goldberg LH. Serious adverse vascular events associated with perioperative interruption of antiplatelet and anticoagulant therapy. Dermatol Surg. 2002;28(11):992-8.

2. Alcalay J, Alkalay R. Controversies in perioperative management of blood thinners in dermatologic surgery: continue or discontinue? Dermatol Surg. 2004;30(8):1091-4.

3. Burger W, Chemnitius JM, Kneissl GD, et al. Low-dose aspirin for secondary cardiovascular prevention - cardiovascular risks after its perioperative withdrawal versus bleeding risks with its continuation - review and meta-analysis. J Intern Med. 2005;257(5):399-414.

4. Bordeaux JS, Martires KJ, Goldberg D, et al. Prospective evaluation of dermatologic surgery complications including patients on multiple antiplatelet and anticoagulant medications. J Am Acad Dermatol. 2011;65(3):576-83.

5. Gerstein NS, Schulman PM, Gerstein WH, et al. Should more patients continue aspirin therapy perioperatively?: clinical impact of aspirin withdrawal syndrome. Ann Surg. 2012;255(5):811-9.

6. Eichhorn W, Kluwe L, Heiland M, et al. Lack of evidence for increased risk of postoperative bleeding after cutaneous surgery in the head and neck in patients taking aspirin. Br J Oral Maxillofac Surg. 2014;52(6):527-9.

7. Dixon AJ, Dixon MP, Dixon JB. Bleeding complications in skin cancer surgery are associated with warfarin but not aspirin therapy. Br J Surg. 2007:94(11):1356-60.

8. Dhiwakar M, Khan NA, McClymont LG. Surgical resection of cutaneous head and neck lesions: does aspirin use increase hemorrhagic risk? Arch Otolaryngol Head Neck Surg. 2006;132(11):1237-41.

Submit your next manuscript to BioMed Central and we will help you at every step:

- We accept pre-submission inquiries

- Our selector tool helps you to find the most relevant journal

- We provide round the clock customer support

- Convenient online submission

- Thorough peer review

- Inclusion in PubMed and all major indexing services

- Maximum visibility for your research 